\title{
Optimal Polarization Synthesis of Arbitrary Arrays With Focused Power Pattern
}

\author{
Benjamin Fuchs, Member, IEEE, and Jean Jacques Fuchs, Member, IEEE
}

\begin{abstract}
The joint synthesis of the spatial power pattern and polarization of arbitrary arrays is addressed. Specifically, the proposed approach gives the solution to a frequently encountered problem, namely the array design (i.e., the determination of the radiating element weightings) to achieve a pattern that is arbitrarily upper bounded, while its polarization is optimized in a given angular region. Any state of polarization (elliptical, circular and linear) can be synthesized and there is no restriction regarding the array geometry and element patterns. The synthesis problem is rewritten as a convex optimization problem, that is efficiently solved using readily available software. This ensures the optimality of the proposed solution. Various numerical results are presented to validate the proposed method and illustrate its potentialities. The synthesis of a sequentially rotated array is first addressed. Then a linear array of equispaced randomly oriented dipoles is considered. Finally, a conformal and a planar array of patches, where the mutual coupling effects are considered, are synthesized to radiate a linear and a circular polarization.
\end{abstract}

Index Terms-Array pattern synthesis, conformal arrays, convex optimization, waveform polarization.

\section{INTRODUCTION}

$\mathbf{E}$ XPLOITING the polarization of a waveform has many advantages. It enables, for instance, to improve the performance of active sensing systems, such as radars [1]. Polarimetric radar systems have thus been developed and efficiently used in various applications [2], [3]. Moreover, the polarization diversity has been shown to improve the performances of communication systems. It indeed significantly increases the capacity of wireless communications [4]. The polarization diversity is also effective to combat fading in mobile wireless communications [5], [6] and to compensate for polarization mismatch due to random handset orientation [7]. Investigations have also been led to show the interest of using polarized arrays for interference rejection in wireless communication systems [8], [9].

If the benefits of using polarized waveforms are well known, the way to generate them, i.e., the synthesis of polarized arrays, has been relatively few reported in the literature. Though the synthesis of antenna arrays has received much attention over the years [10], [11], most of these works focus on spatial power

Manuscript received November 05, 2010; revised April 08, 2011; accepted June 02, 2011. Date of publication August 18, 2011; date of current version December 02, 2011.

B. Fuchs is with the Laboratory of Electromagnetics and Acoustics, Ecole Polytechnique Fédérale de Lausanne, Switzerland on leave from the IETR/University of Rennes I, Rennes Cedex 35042, France (e-mail: b.fuchs@epfl.ch).

J. J. Fuchs is with the IRISA/University of Rennes I, 35042 Rennes Cedex, France (e-mail: fuchs@irisa.fr).

Color versions of one or more of the figures in this paper are available online at http://ieeexplore.ieee.org.

Digital Object Identifier 10.1109/TAP.2011.2165492 pattern synthesis and thus consider that the field emitted by antenna arrays is scalar. These techniques are therefore not able to handle the design of arrays having any elliptical polarization.

It is only quite recently that a few works dealing with the joint synthesis of power pattern and polarization have been reported in the literature. An iterative least square method is presented in [12] to synthesize a main beam with optimized circular polarization for conformal arrays. An analytic approximation of circular polarized patches is used to model the elements radiation pattern. The ideal shape of the far field radiation pattern and the targeted polarization are defined as the desired radiation pattern in a least square optimization procedure.

An adaptive array approach is applied in [13] to a conformal antenna array to synthesize a main beam with optimized polarization employing dual polarized patch antennas as radiating elements.

The aforementioned synthesis methods can not ensure that the optimum is reached, since the optimization problems they solve are not convex or not transformed into convex programs. The exploitation of convexity in array synthesis problems has been introduced by [14] and applied, later on, to various classes of problems as reviewed in [15]. Such a convex formulation has been proposed in [16] to optimally synthesize pencil beams. While the coupling is taken into account, arbitrarily defined upper bounds can be set on the co- and cross-polarization components of the field.

The optimal synthesis of beampattern having any state of polarization via convex optimization has been addressed very recently in [17]-[19] using an array of vector antennas, i.e., antennas composed of orthogonal electric or magnetic dipoles. Specifically, the goal is to find the antenna weightings that achieve a pattern whose main beam pointing at a given direction has a specified polarization, while the sidelobe level is minimized. This vector array synthesis problem is cleverly transformed into a scalar one using an orthogonal transformation, which then makes the problem efficiently solvable.

In this paper, a method to jointly synthesize the spatial power pattern and the polarization of arbitrary arrays is presented. Specifically, the proposed approach designs the array radiating element weightings to achieve a pattern whose power is subject to arbitrary upper bounds, while its polarization is optimized over an angular region. The search for the array element weightings is formulated as a convex optimization problem. This ensures the optimality of the proposed solution that is obtained by transforming the problem into a second order cone program (SOCP) [20]. From now on, it is thus possible to solve this frequently encountered synthesis problem in an efficient way. 


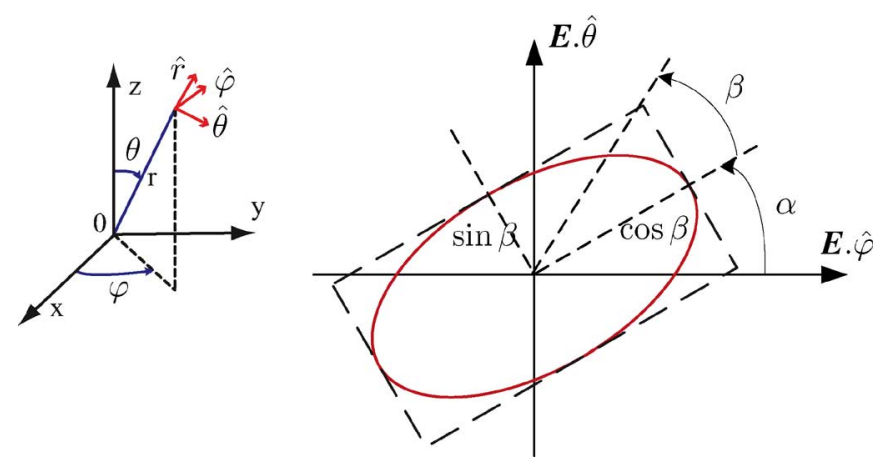

Fig. 1. Coordinate system and polarization ellipse. In far field, the electric field propagates along $\hat{r}$ and lies in a plane orthogonal to $\hat{r}$, that is spanned by $(\hat{\theta}, \hat{\varphi})$. The waveform polarization can be described by the polarization ellipse, with $\alpha$ the orientation angle and $\beta$ the ellipticity angle. $\cos \beta$ and $\sin \beta$ are the major and minor axis lengths of the ellipse respectively.

The paper is organized as follows. In Section II, the synthesis problem is precisely defined, formulated as a convex optimization problem and transformed into a SOCP ready to be solved. Various numerical results are presented in Section III to both validate and illustrate the potentialities of the proposed synthesis method. Conclusions are drawn in Section IV.

\section{PROBLEM FORMULATION AND RESOLUTION}

\section{A. Antenna Array}

Let us consider an antenna array composed of $N$ elements placed at arbitrary but known locations $\vec{r}_{i}$ with $i=1, \ldots, N$. The problem is described for a one-dimensional pattern synthesis. This synthesis is performed over the polar angle $\theta$ in a fixed azimuthal plane $\varphi=\varphi_{0}$ (see Fig. 1), that is omitted in the notations. The extension to a two-dimensional (2-D) pattern synthesis, i.e., a synthesis over both angular directions $\theta$ and $\varphi$, is straightforward and two examples of 2-D pattern synthesis are shown in Sections III.A and III.D.

The array factor $\boldsymbol{g}$ in the direction $\theta$ is:

$$
\boldsymbol{g}(\theta)^{H}=\left[e^{-j k \vec{r}_{1} \cdot \vec{r}(\theta)} \ldots e^{-j k \vec{r}_{i} \cdot \vec{r}(\theta)} \ldots e^{-j k \vec{r}_{N} \cdot \vec{r}(\theta)}\right]
$$

where $\cdot{ }^{H}$ denotes the Hermitian transposition, $k$ is the free space wave number and $\vec{r}(\theta)$ is the unit vector in the direction $\theta$ (and azimuthal plane $\left.\varphi_{0}\right)$.

The element $i$ of the array radiates a vectorial far field pattern $f_{i}(\theta)$, which has, in general, both a $\theta$ and $\varphi$ component: $f_{i}(\theta)=$ $\left[f_{\theta_{i}}(\theta) \quad f_{\varphi_{i}}(\theta)\right]$. This leads to the following vectors:

$$
\begin{aligned}
\boldsymbol{f}_{\theta}(\theta)^{H} & =\left[f_{\theta 1}(\theta) \ldots f_{\theta N}(\theta)\right] \\
\boldsymbol{f}_{\varphi}(\theta)^{H} & =\left[f_{\varphi_{1}}(\theta) \ldots f_{\varphi_{N}}(\theta)\right] .
\end{aligned}
$$

Note that arbitrary arrays can be considered, since there is no restriction regarding the array geometry and the element patterns. The latter can indeed be obtained from either analytical formula, simulation results or measured data.

The vectorial antenna array response is:

$$
\begin{array}{ll}
\boldsymbol{a}_{\theta}(\theta)=\boldsymbol{g}(\theta) \cdot * \boldsymbol{f}_{\theta}(\theta) & \in C^{N \times 1} \\
\boldsymbol{a}_{\varphi}(\theta)=\boldsymbol{g}(\theta) \cdot * \boldsymbol{f}_{\varphi}(\theta) & \in C^{N \times 1}
\end{array}
$$

where $\boldsymbol{u} \cdot * \boldsymbol{v}$ is the term-by-term product of the vectors $\boldsymbol{u}$ and $\boldsymbol{v}$.

The electric field radiated by the array is:

$$
\boldsymbol{E}(\theta)=\left[\begin{array}{l}
E_{\theta}(\theta) \\
E_{\varphi}(\theta)
\end{array}\right]=\left[\begin{array}{l}
\boldsymbol{a}_{\theta}(\theta)^{H} \boldsymbol{w} \\
\boldsymbol{a}_{\varphi}(\theta)^{H} \boldsymbol{w}
\end{array}\right]
$$

where $\boldsymbol{w}$ is the $N$-dimensional vector of complex element weightings. The weighting vector $\boldsymbol{w}$ is thus composed of $N$ scalars $w_{i}$ that control the vectorial field $\boldsymbol{f}_{i}(\theta)$ radiated by the array elements. These complex weightings $w_{i}$ are the unknowns to determine.

\section{B. Waveform Polarization}

A brief reminder on the waveform polarization is presented to introduce the notations that are used later.

The polarization of an electric field can be defined, see [21], by the orientation angle $\alpha$ and the ellipticity angle $\beta$ represented in Fig. 1. For instance, $\beta=0$ and $\beta= \pm \pi / 4$ yield linear and circular polarizations respectively. Moreover, the axial ratio denoted AR, often used to evaluate the quality of the circular wave polarization, is equal to:

$$
\mathrm{AR}=\frac{\text { major ellipse axis length }}{\text { minor ellipse axis length }}=\frac{\cos \beta}{\sin \beta} .
$$

It must be pointed out, that there is a one-to-one relationship between the angles $(\alpha, \beta)$ and the coefficients $(\gamma, \delta)$ of the ratio $\left(E_{\varphi}\right) /\left(E_{\theta}\right)=\gamma e^{j \delta}$ if $E_{\theta}^{2}+E_{\varphi}^{2} \neq 0$, as demonstrated in [22]. Specifically, $(\alpha, \beta)$ is linked to $(\gamma, \delta)$ by:

$$
\begin{aligned}
\cos 2 \alpha & =\frac{1-\gamma^{2}}{\sqrt{1+\gamma^{4}+2 \gamma^{2} \cos (2 \gamma)}} \\
\sin 2 \alpha & =\frac{-2 \gamma \cos \delta}{\sqrt{1+\gamma^{4}+2 \gamma^{2} \cos (2 \gamma)}} \\
\beta & =\arcsin \frac{\sqrt{1+\frac{2 \gamma}{1+\gamma^{2}} \sin \delta}-\sqrt{1-\frac{2 \gamma}{1+\gamma^{2}} \sin \delta}}{2}
\end{aligned}
$$

where $\alpha=[-\pi / 2, \pi / 2]$ and $\beta=[-\pi / 4, \pi / 4]$.

In the sequel, the waveform polarization is defined using $(\gamma, \delta)$.

\section{Array Pattern Synthesis}

The array pattern synthesis, performed over the polar angle $\theta$ in a plane $\varphi=\varphi_{0}$ (omitted for clearness reasons), is now detailed. The problem amounts to find the weighting vector $\boldsymbol{w}$ in (6) to achieve a pattern that has both spatial power and polarization requirements. More precisely, the spatial power is taken care of by constraints whereas the polarization is the optimization goal. As represented in Fig. 2, the goal is to synthesize a pattern having:

- a main beam in the direction $\theta_{0}$ with sidelobes below a given upper bound $\rho(\theta)$ over an angular region $S$ and

- a wave polarization, characterized by $\left(\gamma_{0}, \delta_{0}\right)$, optimized over an angular region $P$.

The regions $P$ and $S$ can overlap.

The magnitude of the vector electric field is equal to:

$$
\|\boldsymbol{E}(\theta)\|_{2}=\left[\left|E_{\theta}(\theta)\right|^{2}+\left|E_{\varphi}(\theta)\right|^{2}\right]^{1 / 2} .
$$




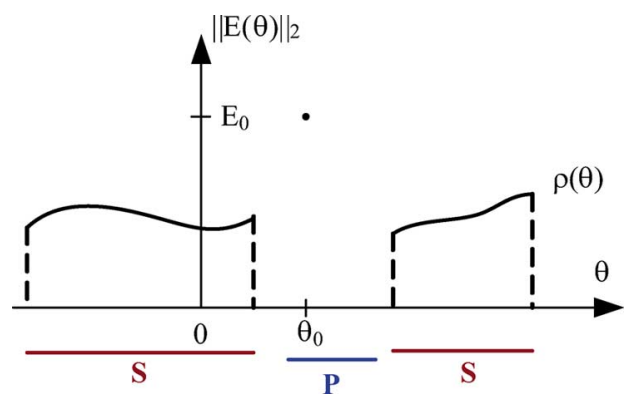

Fig. 2. Schematic view of the pattern synthesis problem in a plane $\varphi=\varphi_{0}$. The magnitude of the electric field must reach $E_{0}$ at the direction $\theta_{0}$ and remain below an arbitrary upper bound $\rho(\theta)$ over the angular region $S$ while the polarization $\left(\gamma_{0}, \delta_{0}\right)$ is optimized over $P$.

A unique, potentially $\theta$-dependent, bound $\rho(\theta)$ will be imposed on this quantity over $S$ to handle the spatial power constraint.

Let us now focus on the polarization constraint. A given polarization $\left(\gamma_{0}, \delta_{0}\right)$ can be synthesized over a range of directions $(\theta \in P)$ by imposing:

$$
\left|\frac{E_{\varphi}(\theta)}{E_{\theta}(\theta)}-\gamma_{0} e^{j \delta_{0}}\right| \leq \epsilon, \quad \forall \theta \in P
$$

where $\epsilon$ allows to tune the degree of accuracy with which the polarization is achieved.

Since relation (9) is difficult to enforce, it is replaced by:

$$
\begin{array}{r}
\left|E_{\varphi}(\theta)-\gamma_{0} e^{j \delta_{0}} E_{\theta}(\theta)\right| \leq \epsilon^{\prime}(\theta), \quad \forall \theta \in P \\
\text { with } \epsilon^{\prime}(\theta)=\epsilon\left|E_{\theta}(\theta)\right| .
\end{array}
$$

In order to guaranty a constant upper bound $\epsilon$ over $P$ in (9), one has to enforce a $\theta$-dependent $\epsilon^{\prime}(\theta)$ in (10). Since the value of $\left|E_{\theta}(\theta)\right|$ is not a priori known, it will be replaced by an estimate denoted $\left|E_{\theta}^{-}(\theta)\right|$. The synthesis problem will thus be solved iteratively and $\left|E_{\theta}^{-}(\theta)\right|$ in the right member of the first constraint is replaced by $E_{0} /\left[1+\gamma_{0}^{2}\right]^{1 / 2}$ at the first iteration and by the outcome of the previous iteration afterwards. Convergence generally occurs after three iterations.

The synthesis problem described in Fig. 2 can be written as:

$$
\begin{aligned}
& \min _{\boldsymbol{w}} \epsilon \\
& \text { under } \begin{cases}\left|E_{\varphi}(\theta)-\gamma_{0} e^{j \delta_{0}} E_{\theta}(\theta)\right| \leq \epsilon\left|E_{\theta}^{-}(\theta)\right|, & \forall \theta \in P \\
{\left[\left|E_{\theta}(\theta)\right|^{2}+\left|E_{\varphi}(\theta)\right|^{2}\right]^{1 / 2} \leq \rho(\theta),} & \forall \theta \in S \\
\Re\left\{E_{\theta}\left(\theta_{0}\right)\right\}=E_{0} /\left[1+\gamma_{0}^{2}\right]^{1 / 2} & \end{cases}
\end{aligned}
$$

To justify the last constraint, one observes that if the polarization of the electric field is equal to $\left(\gamma_{0}, \delta_{0}\right)$ at the direction $\theta_{0}$, i.e., $E_{\varphi}\left(\theta_{0}\right)=\gamma_{0} e^{j \delta_{0}} E_{\theta}\left(\theta_{0}\right)$, then the magnitude of the total electric field is: $\left[\left|E_{\theta}\left(\theta_{0}\right)\right|^{2}+\left|E_{\varphi}\left(\theta_{0}\right)\right|^{2}\right]^{1 / 2}=\left|E_{\theta}\left(\theta_{0}\right)\right|\left[1+\gamma_{0}^{2}\right]^{1 / 2}$. Fixing then $\Re\left\{E_{\theta}\left(\theta_{0}\right)\right\}=E_{0} /\left[1+\gamma_{0}^{2}\right]^{1 / 2}$, one expects that the optimum in $\epsilon$ will yield a $\boldsymbol{w}$ that is such that $\Im\left\{E_{\theta}\left(\theta_{0}\right)\right\}=0$, i.e., such that the phase of $E_{\theta}$ is zero at $\theta_{0}$. Imposing this condition on $\boldsymbol{w}$ is not a limitation, since $\boldsymbol{w}$ is defined up to an arbitrary phase.
With the notations of (6), one gets:

$$
\begin{array}{ll}
\min _{\boldsymbol{w}} \epsilon & \operatorname{lnder} \begin{cases}\left|\boldsymbol{a}_{\varphi}(\theta)^{H} \boldsymbol{w}-\gamma_{0} e^{j \delta_{0}} \boldsymbol{a}_{\theta}(\theta)^{H} \boldsymbol{w}\right| \leq \epsilon\left|E_{\theta}^{-}(\theta)\right|, & \forall \theta \in P \\
{\left[\left|\boldsymbol{a}_{\theta}(\theta)^{H} \boldsymbol{w}\right|^{2}+\left|\boldsymbol{a}_{\varphi}(\theta)^{H} \boldsymbol{w}\right|^{2}\right]^{1 / 2} \leq \rho(\theta),} & \forall \theta \in S . \\
\Re\left\{\boldsymbol{a}_{\theta}\left(\theta_{0}\right)^{H} \boldsymbol{w}\right\}=E_{0} /\left[1+\gamma_{0}\right]^{1 / 2} & \end{cases}
\end{array}
$$

To further formalize (12), the constraints on the sidelobe level and beam polarization are discretized. One introduces $M$ directions $\left\{\theta_{m}\right\}, m=1, \ldots, M$ and $K$ directions $\left\{\theta_{k}\right\}, k=$ $M+1, \ldots, M+K$ that covers $P$ and $S$ respectively.

The synthesis problem (12) becomes as follows:

$$
\begin{aligned}
& \min _{\boldsymbol{w}} \epsilon \\
& \operatorname{under}\left\{\begin{array}{l}
\left|E_{\varphi_{m}}-\gamma_{0} e^{j \delta_{0}} E_{\theta_{m}}\right| \leq \epsilon\left|E_{\theta_{m}}^{-}\right|, \\
\text {for } m=1, \ldots, M \\
{\left[\left|E_{\theta_{k}}\right|^{2}+\left|E_{\varphi_{k}}\right|^{2}\right]^{1 / 2} \leq \rho_{k},} \\
\text { for } k=M+1, \ldots, M+K \\
\Re\left\{E_{\theta_{0}}\right\}=E_{0} /\left[1+\gamma_{0}^{2}\right]^{1 / 2}
\end{array}\right.
\end{aligned}
$$

where $E_{\varphi_{i}}=\boldsymbol{a}_{\varphi}\left(\theta_{i}\right)^{H} \boldsymbol{w}, E_{\theta_{i}}=\boldsymbol{a}_{\theta}\left(\theta_{i}\right)^{H} \boldsymbol{w}, \rho_{k}=\rho\left(\theta_{k}\right)$ and where the expression of $\left|E_{\theta_{m}}^{-}\right|$in terms of $E_{0}$ or the previous optimal $\boldsymbol{w}$ vector is not detailed.

The optimization problem (13) is convex, which ensures the optimality of the design solution. It can be transformed, as detailed in the next Section, into a Second Order Cone Program (SOCP) [20], that is solved iteratively, as described below relation (10). Convergence generally occurs after a few (typically three) iterations.

\section{Resolution via Second Order Cone Program (SOCP)}

A SOCP has the following standard form:

$$
\min \boldsymbol{c}^{T} \boldsymbol{x} \quad \text { under } \boldsymbol{B} \boldsymbol{x}=\boldsymbol{b}, \quad \boldsymbol{x} \in K
$$

where $K$ represents second order cones. A second order cone $L_{p}$ of dimension $p$ is of the form:

$$
\begin{aligned}
L_{p} & =\left\{\boldsymbol{x} \mid x_{1} \geq\left\|x_{2}, x_{3}, \ldots, x_{p}\right\|_{2}\right\} \\
& =\left\{\boldsymbol{x} \mid x_{1} \geq\left(\sum_{i=2}^{p} x_{i}^{2}\right)^{1 / 2}\right\} .
\end{aligned}
$$

A SOCP can be seen as a generalization of a linear program. It has been established [20] that one can extend the linear program (theory and algorithms) to the conic program.

To handle the polarization constraint, one introduces the complex number $z_{m}=E_{\varphi_{m}}-\gamma_{0} e^{j \delta_{0}} E_{\theta m}$ and associates a triplet $\left\{p_{m}, \Re\left(z_{m}\right), \Im\left(z_{m}\right)\right\} \in L_{3}$, for $m=1$ to $M$. It yields $p_{m} \geq\left|z_{m}\right|=\left[\Re\left(z_{m}\right)^{2}+\Im\left(z_{m}\right)^{2}\right]^{1 / 2}$ and one has consequently $\left|z_{m}\right| \leq p_{m} \leq \epsilon$, for $m=1$ to $M$.

The sidelobe level constraint $\left[\left|E_{\theta k}\right|^{2}+\left|E_{\varphi_{k}}\right|^{2}\right]^{1 / 2} \leq \rho_{k}$ in (13), is taken into account by creating a second order cone of 
dimension 5: $\left\{s_{k}, \Re\left(E_{\theta k}\right), \Im\left(E_{\theta k}\right), \Re\left(E_{\varphi_{k}}\right), \Im\left(E_{\varphi_{k}}\right)\right\} \in L_{5}$. It implies that:

$$
s_{k} \geq\left[\Re\left(E_{\theta k}\right)^{2}+\Im\left(E_{\theta k}\right)^{2}+\Re\left(E_{\varphi_{k}}\right)^{2}+\Im\left(E_{\varphi_{k}}\right)^{2}\right]^{1 / 2}
$$

which leads to $\left\|E_{k}\right\|_{2} \leq s_{k} \leq \rho_{k}$ for $k=M+1, \ldots, M+K$.

Standard transformations, namely the use of slack variables, are then used to rewrite the inequalities of (11) into equalities, as required in (14).

Note that the use of SOCP in (16) allows to constrain precisely the magnitude of the vector field:

$$
\max _{\theta \in S}\|E(\theta)\|_{2}=\max _{\theta \in S}\left[\left|E_{\theta}(\theta)\right|^{2}+\left|E_{\varphi}(\theta)\right|^{2}\right]^{1 / 2} \leq \rho .
$$

This is indeed a much weaker constraint than imposing, as done in [13], [16], an upper bound on the two components of the field, e.g., $\max _{\theta \in S}\left|E_{\theta}(\theta)\right| \leq \rho_{1}$ and $\max _{\theta \in S}\left|E_{\varphi}(\theta)\right| \leq \rho_{2}$, with $\rho_{1}^{2}+\rho_{2}^{2}=\rho^{2}$.

Once the possibly delicate transcription of the synthesis problem into a SOCP (as described above) has been performed, the existence of free software that solve efficiently SOCP, without any further tuning, justifies largely its use. To solve the SOCP (14), the optimization toolbox SeDuMi [25] is used. More examples of synthesis problems using SOCP are given in [23], [24].

\section{NUMERICAL RESULTS}

In this Section, numerical results are presented to validate and illustrate the potentialities of the proposed approach. Let us remind that any state of polarization $\left(\gamma_{0}, \delta_{0}\right)$ in (10) can be synthesized, but in the examples below, only linear and circular polarization are considered. They corresponds respectively to the minimization of $\left|E_{\varphi}\right|$ and $\left|E_{\varphi}-j E_{\theta}\right|$. In the case of the linear polarization synthesis, relation (10) becomes then $\left|E_{\varphi}\right| \leq$ $\epsilon$ and the synthesis procedure requires therefore no iteration.

First, a sequentially rotated array composed of dipoles is designed to radiate a circularly polarized wave in order to validate the method. Then, a linear array of dipoles is synthesized to radiate a pattern with a spatial power constraint and an optimized polarization. The synthesis of a conformal array, where the coupling effects are taken into account, is also presented. Finally, a two-dimensional (2-D) pattern synthesis problem is addressed for a planar array composed of randomly oriented patches.

\section{A. Validation: Sequentially Rotated Array}

The approach, described in Section II, is here extended in a straightforward way to the synthesis of 2-D patterns. A $\varphi$ dependency has to be considered in the (1) to (13).

It is well known that sequentially rotated arrays of linearly polarized elements can generate circularly polarized radiation [26], [27]. Let us consider such an array composed of 16 dipoles, as represented in Fig. 3. This array is synthesized to radiate a pattern having 2-D spatial power constraints:

- a main beam in the broadside direction $\theta_{0}=0^{\circ}$, i.e., $\left(u_{0}, v_{0}\right)=(0,0)$ since $u=\sin \theta \cos \varphi$ and $v=\sin \theta \sin \varphi$, with sidelobe levels $\rho(\theta, \varphi)$ below

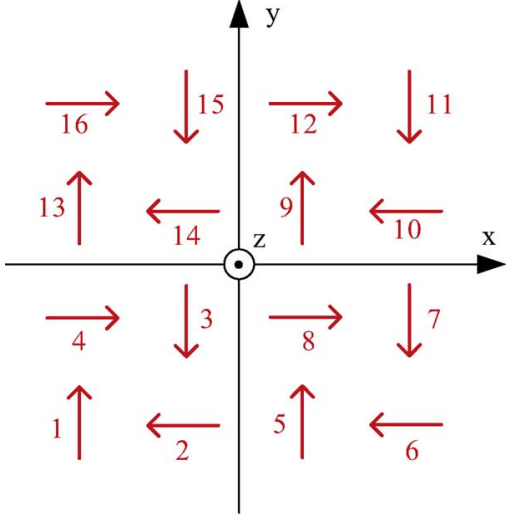

Fig. 3. Schematic view of the sequentially rotated array composed of 16 dipoles spaced by $\lambda_{0} / 2$.

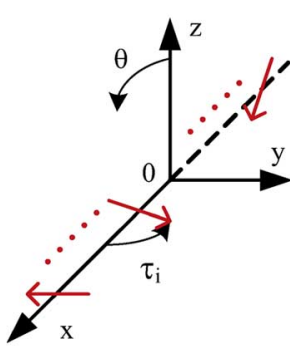

(a)

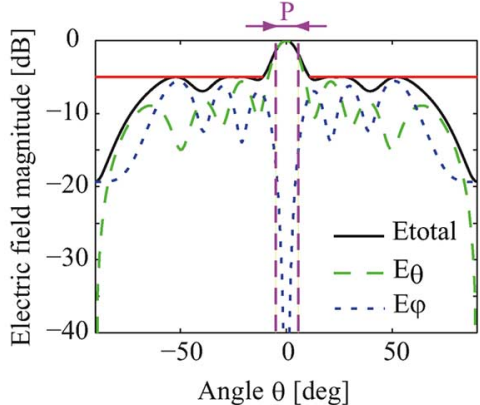

(b)
Fig. 4. Results of the circular polarization synthesis with a sequentially rotated array composed of 16 dipoles. (a) The magnitude of the total electric field is lower than $12 \mathrm{~dB}$ outside the white circle. The axial ratio on both axis $u=0$ and $v=0$ is plotted, when optimized over (b) the broadside direction (i.e., $\mathrm{P}=0^{\circ}$ ) and (c) the angular region $\mathrm{P}=\left[-25^{\circ}, 25^{\circ}\right]$, i.e., for $u$ or $v \in[-0.4,0.4]$.

$-12 \mathrm{~dB}$ for $\theta \geq 25^{\circ}$, i.e., outside the white circle in Fig. 4(a)

and a circular polarization:

- optimized either in the broadside direction $P=\theta_{0}=0^{\circ}$ or over the angular range $P=\theta \leq 25^{\circ}$.

The synthesized far field pattern and axial ratio are plotted in Fig. 4. While the power pattern complies to the requirements, the axial ratio plots, Fig. 4(b),(c), clearly show that a circular polarization is synthesized over the region $P$. The optimized weightings are given in Table I. The 16-element array can be seen as 4 sub-arrays composed of 4 sequentially rotated dipoles, namely $(1,6,11,16),(2,7,12,13),(3,8,9,14)$ and $(4,5$, $10,15)$. Within each sub-array, the optimized weightings have almost the same magnitude and a phase shift close to $90^{\circ}$, which is consistent with the results shown in [26], [27].

\section{B. Linear Arrays}

1) Linear Polarization Synthesis: A linear array of ten equispaced dipoles, that are randomly oriented, is considered to synthesize a pattern having a broadside main beam, sidelobe levels below $-5 \mathrm{~dB}$ for $\theta$ outside $\left[-10^{\circ}, 10^{\circ}\right]$ and an optimized linear polarization over $P=\left[-5^{\circ}, 5^{\circ}\right.$. A schematic view of the array is represented in Fig. 5(a) and the dipoles positions and specific 
TABLE I

Optimal WeIGHTINGS OF THE SEQUENTIALly Rotated ARRAY SyNTHESIS

\begin{tabular}{ccccc}
\hline \hline & \multicolumn{2}{c}{$\mathrm{P}=0^{\circ}$} & \multicolumn{2}{c}{$\mathrm{P}=\left[-25^{\circ}, 25^{\circ}\right]$} \\
feed & $|w|$ & $\angle w[\mathrm{deg}]$ & $|w|$ & $\angle w[\mathrm{deg}]$ \\
\hline 1 & 0.42 & 168 & 0.59 & -173 \\
2 & 0.65 & -85 & 0.95 & -90 \\
3 & 1.00 & 0 & 1.00 & -4 \\
4 & 0.65 & 94 & 0.85 & 90 \\
\hline 5 & 0.66 & -176 & 0.84 & -180 \\
6 & 0.41 & -103 & 0.59 & -83 \\
7 & 0.65 & 4 & 0.96 & 0 \\
8 & 1.00 & 90 & 1.00 & 86 \\
\hline 9 & 1.00 & 180 & 1.00 & 176 \\
10 & 0.65 & -86 & 0.85 & -90 \\
11 & 0.42 & -12 & 0.59 & 7 \\
12 & 0.65 & 94 & 0.95 & 90 \\
\hline 13 & 0.65 & -176 & 0.96 & 180 \\
14 & 1.00 & -90 & 1.00 & -94 \\
15 & 0.66 & 4 & 0.84 & 0 \\
16 & 0.41 & 77 & 0.59 & 97 \\
\hline \hline
\end{tabular}

orientations together with the optimal weightings are given in Table II.

The synthesized far field patterns are shown in Fig. 5(b). While the magnitude of the total field complies to the sidelobe level constraint, $\left|E_{\varphi}\right|$ is at least $16.8 \mathrm{~dB}$ lower than $\left|E_{\theta}\right|$ over $P$, which confirms the linear polarization of the wave.

2) Circular Polarization Synthesis: A linear array of ten crossed dipoles, that are randomly oriented and spaced by $0.75 \lambda_{0}$, is considered to synthesize a main beam in the direction $\theta_{0}=10^{\circ}$ with an optimized circular polarization over $P=\left[-10^{\circ}, 30^{\circ}\right]$. The sidelobe levels are constrained to remain below $-10 \mathrm{~dB}$ over $S=\left[-90^{\circ}, 0^{\circ}\right] \cup\left[20^{\circ}, 90^{\circ}\right]$. A schematic view of the array is represented in Fig. 6(a) and the dipoles positions and orientations are given in Table III.

The synthesized far field patterns Fig. 6(b) show that the spatial power constraints are well respected. Moreover, the $\theta$ and $\varphi$ components of the field have the same magnitude over $P$. This is confirmed by the axial ratio, plotted in Fig. 6(c), that is lower than $0.1 \mathrm{~dB}$ over $P$, which establishes the good quality of the synthesized circular polarization.

\section{Conformal Array}

An array of five patches that are conformed on a cylinder, as represented in Fig. 7(a), is considered. Each square patch $i$ is fed by two coaxial probes $i X$ and $i Y$, as shown in Fig. 7(b). Each element is therefore dually polarized.

The goal is to find the complex weightings of each coaxial probe in order to radiate a main beam in the direction $\theta_{0}=10^{\circ}$ with sidelobe levels below $-10 \mathrm{~dB}$ for $\theta \in S=\left[-90^{\circ},-3^{\circ}\right] \cup\left[23^{\circ}, 90^{\circ}\right]$ and an optimized circular polarization over $P=\left[0^{\circ}, 20^{\circ}\right]$.

The active element pattern method [28] is applied to calculate the pattern of the fully excited array. Each patch is simulated in the array environment with a full wave numerical software (Ansoft HFSS) to provide the array response $\boldsymbol{a}_{\theta}$ and $\boldsymbol{a}_{\varphi}$ of (6). Using this method enables one to take the mutual coupling effects into account.

The optimal weightings of the conformal array synthesis problem are given in Table IV. The synthesized far field patterns

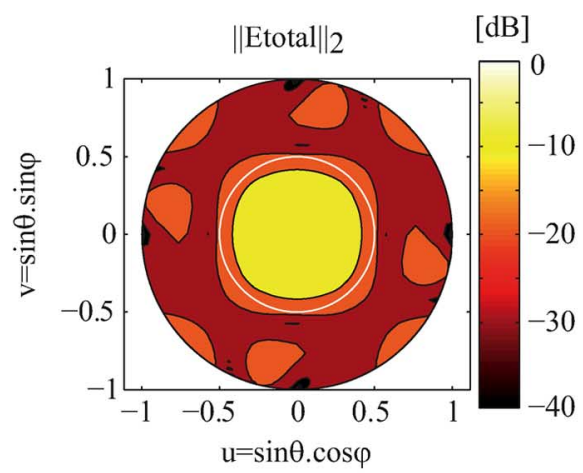

(a)

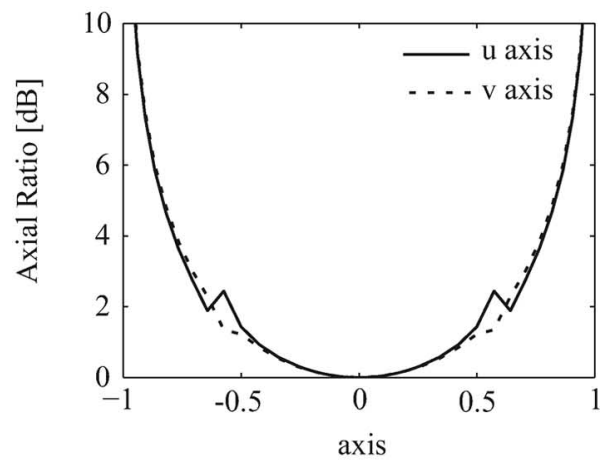

(b)

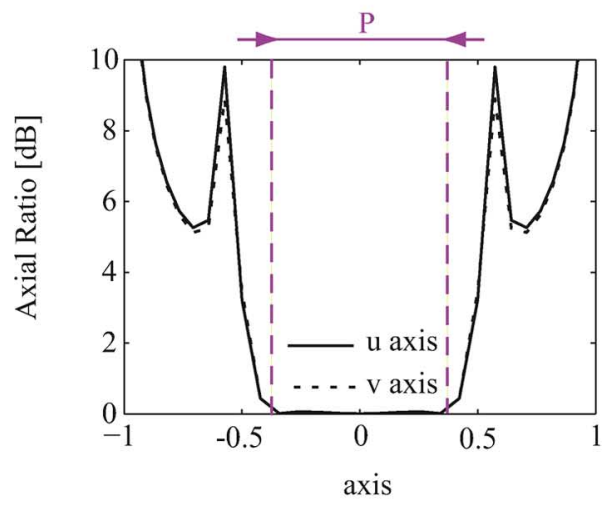

(c)

Fig. 5. (a) Schematic view of the linear array composed of 10 dipoles that are randomly oriented (see angle $\tau_{i}$ ) in the (xOy) plane and spaced by $0.5 \lambda_{0}$. (b) Far field patterns of the linear polarization synthesis.

TABLE II

SETtings AND RESUlts OF THE LINEAR POLARIZATION SyNTHESIS With A LINEAR ARRAY OF DIPOLES

\begin{tabular}{ccc}
\hline \hline position & orientation & weighting \\
{$[\lambda]$} & $\tau[\mathrm{deg}]$ & $w$ \\
\hline-2.25 & 280 & 0.02 \\
-1.75 & 150 & -0.93 \\
-1.25 & 340 & 0.02 \\
-0.75 & 30 & 1.00 \\
-0.25 & 60 & -0.11 \\
0.25 & 250 & -0.26 \\
0.75 & 70 & -0.02 \\
1.25 & 10 & 0.59 \\
1.75 & 30 & 0.03 \\
2.25 & 70 & -0.29 \\
\hline \hline
\end{tabular}

and the resulting axial ratio are plotted in Fig. 7(c),(d). A very 


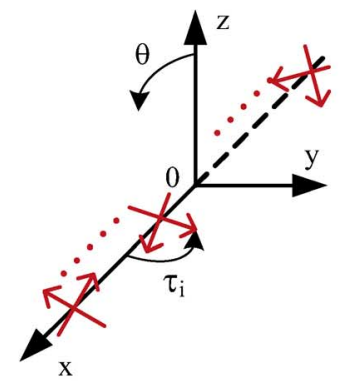

(a)

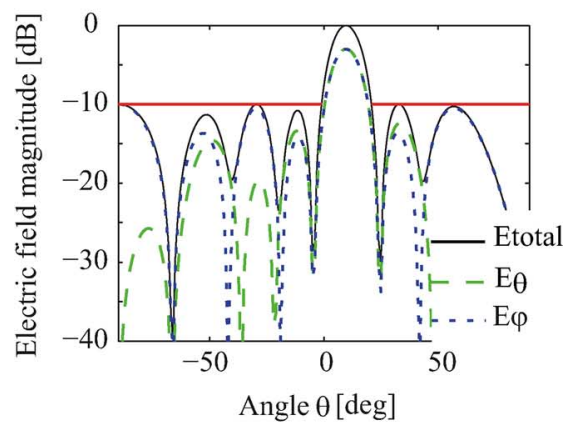

(b)

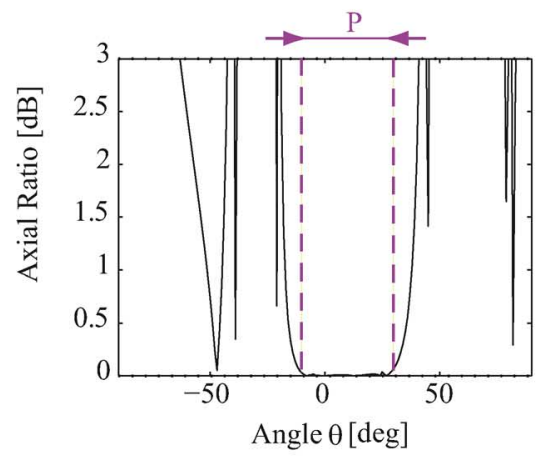

(c)

Fig. 6. (a) Schematic view of the linear array composed of 10 crossed dipoles that are randomly oriented (see angle $\tau_{i}$ ) in the (xOy) plane and spaced by $0.75 \lambda_{0}$. Results of the circular polarization synthesis: (b) the normalized far field patterns and (c) the axial ratio.

TABLE III

SetTings and Results OF THE CirCUlar Polarization SyNTHESIS With A LINEAR ARRAY OF CROSSED DIPOLES

\begin{tabular}{cccc}
\hline \hline position & orientation & \multicolumn{2}{c}{ weighting } \\
{$[\lambda]$} & $\tau[\mathrm{deg}]$ & $|w|$ & $\angle w[\mathrm{deg}]$ \\
\hline-1.5 & 50 & 1.00 & -126 \\
-1.5 & $90+50$ & 0.93 & -38 \\
-0.75 & 120 & 0.78 & -99 \\
-0.75 & $90+120$ & 0.83 & -22 \\
0 & 70 & 0.78 & 164 \\
0 & $90+70$ & 0.62 & -116 \\
0.75 & 210 & 0.86 & -109 \\
0.75 & $90+210$ & 0.74 & -9 \\
1.5 & 330 & 0.98 & -35 \\
1.5 & $90+330$ & 0.95 & 59 \\
\hline \hline
\end{tabular}

good circular polarization is obtained over $P$ and the sidelobe level respects the requirements.

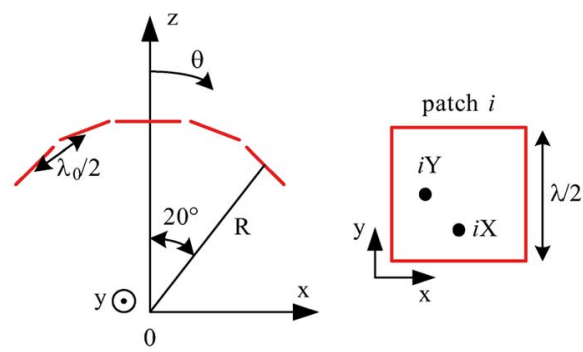

(a)

(b)

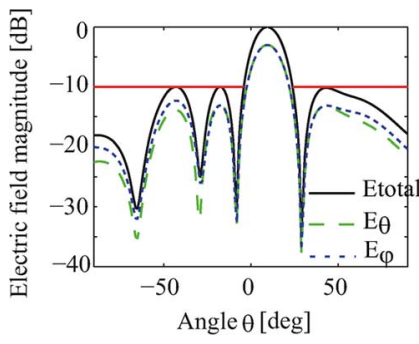

(c)

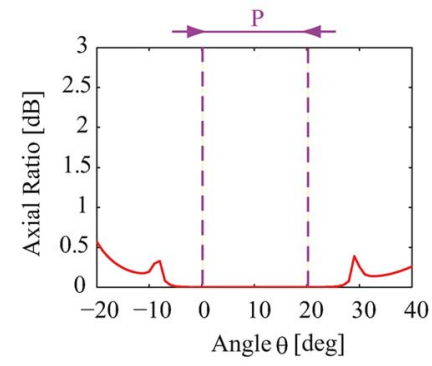

(d)
Fig. 7. (a) Schematic view of the conformal array composed of five patches. (b) Top view of the dual polarized square patch $i$ fed by two coaxial probes $i X$ and $i Y$. Synthesized circular polarization with (c) the electric field magnitude and (d) the axial ratio.

TABLE IV

OPtimal Weightings OF THE CONFORMAL ARRAY SyNTHESIS PROBLEM

\begin{tabular}{ccc}
\hline \hline feed & $|w|$ & $\angle w[\mathrm{deg}]$ \\
\hline $1 \mathrm{X}$ & 0.41 & 32 \\
$1 \mathrm{Y}$ & 0.47 & 121 \\
$2 \mathrm{X}$ & 0.34 & -133 \\
$2 \mathrm{Y}$ & 0.21 & -39 \\
$3 \mathrm{X}$ & 0.66 & -31 \\
$3 \mathrm{Y}$ & 0.65 & 58 \\
$4 \mathrm{X}$ & 0.18 & 134 \\
$4 \mathrm{Y}$ & 0.13 & -145 \\
$5 \mathrm{X}$ & 1.00 & -13 \\
$5 \mathrm{Y}$ & 0.84 & -102 \\
\hline \hline
\end{tabular}

\section{Planar Array for 2-D Pattern Synthesis}

A planar array is used to synthesize a 2-D pattern having spatial power constraints and an optimized polarization. This array is composed of $3 \times 3$ dual polarized patches with a random orientation, as represented in Fig. 8(a). The active element pattern method, described in Section III.C, is applied to calculate the array pattern radiation in order to consider the mutual coupling effects.

1) Circular Polarization Synthesis: The synthesis problem is the following:

- a main beam in the direction $\left(u_{0}, v_{0}\right)=(0.2,0.3)$, i.e., $\left(\theta_{0}, \varphi_{0}\right)=\left(21^{\circ}, 56^{\circ}\right)$, with an upper bound $\rho(\theta, \varphi)$ of -10 dB outside the circle in Fig. 8(b) and

- a circular polarization optimized inside the circle in Fig. 8(c).

The contour plots Fig. 8(b) show that the spatial power constraint is respected. The axial ratio, represented in Fig. 8(c), is lower than $0.2 \mathrm{~dB}$ inside the circle where the polarization has been optimized.

The computation time of such synthesis problem (the matrix $B$ in (14) is of dimension larger than $1300 \times 1600$ for a number 


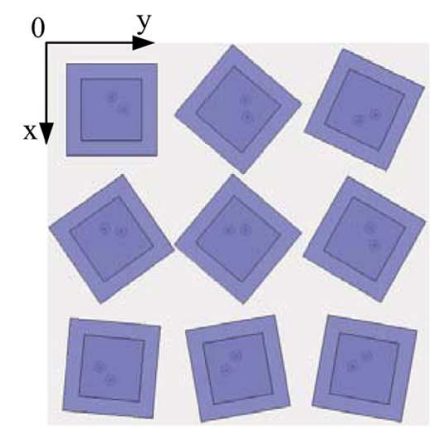

(a)

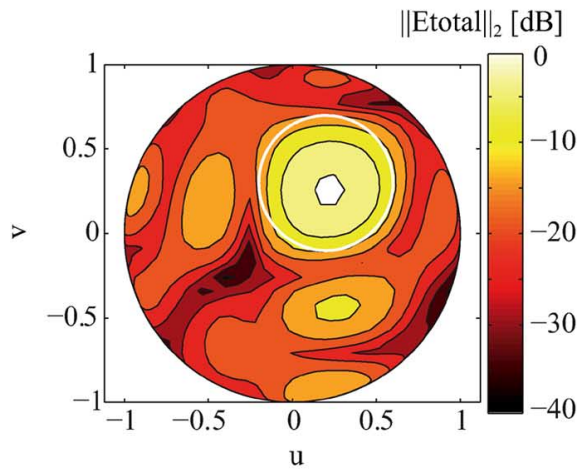

(b)

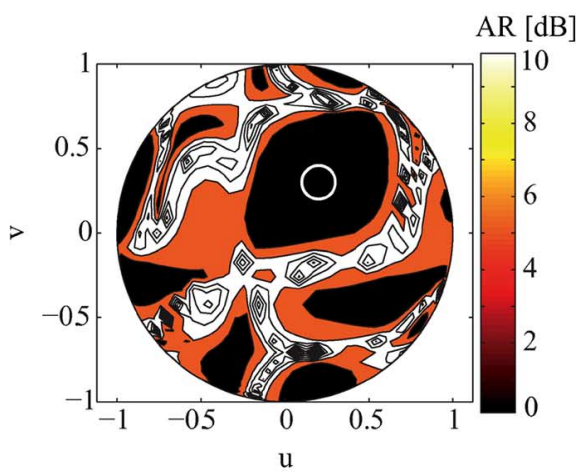

(c)

Fig. 8. (a) Top view of the planar array composed of $3 \times 3$ dual polarized patches with random orientation. (b) Contour plot of the synthesized total electric field. The sidelobe levels are below $-10 \mathrm{~dB}$ outside the white circle. (c) Contour plot of the axial ratio. A circular polarization has been optimized inside the white circle, where AR is lower than $0.2 \mathrm{~dB}$.

of unknowns $\boldsymbol{w}$ equal to 18 ) is less than 5 seconds on a standard laptop.

2) Linear Polarization Synthesis: For this synthesis problem, the spatial power constraints are unchanged $\left(u_{0}, v_{0}\right)=(0.2,0.3)$ and $\rho(\theta, \varphi)=-10 \mathrm{~dB}$ outside the circle represented in Fig. 9(a). A linear polarization according to $\theta$ is optimized inside the circle plotted in Fig. 9(b),(c). The contour plots of the synthesized patterns confirm that, in this region, $\left|E_{\varphi}\right|$ is at most $19 \mathrm{~dB}$ lower than $\left|E_{\theta}\right|$.

\section{CONCLUSION}

A synthesis method to design arrays that radiate a pattern, having both spatial power constraints (upper bounded sidelobes levels) and an optimized specified polarization over a given angular range, has been proposed. Arbitrary arrays, i.e., arrays of

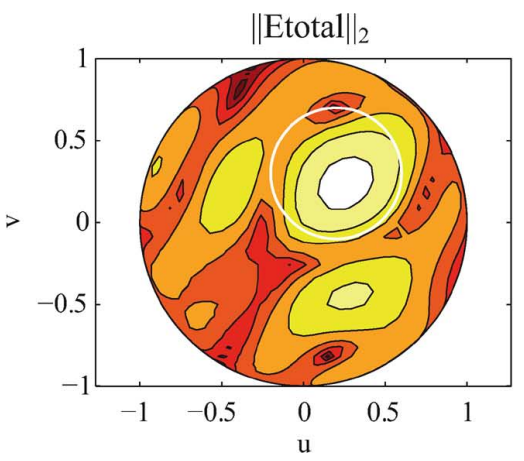

(a)

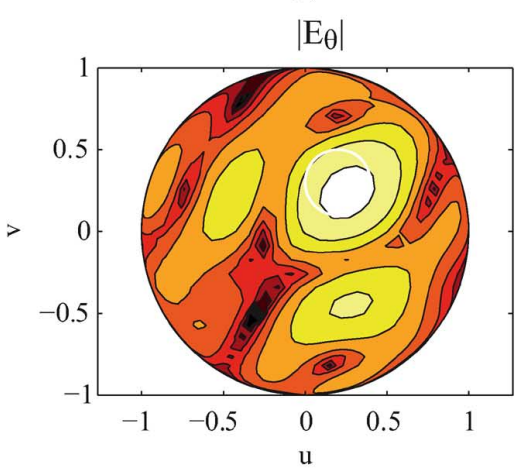

(b)

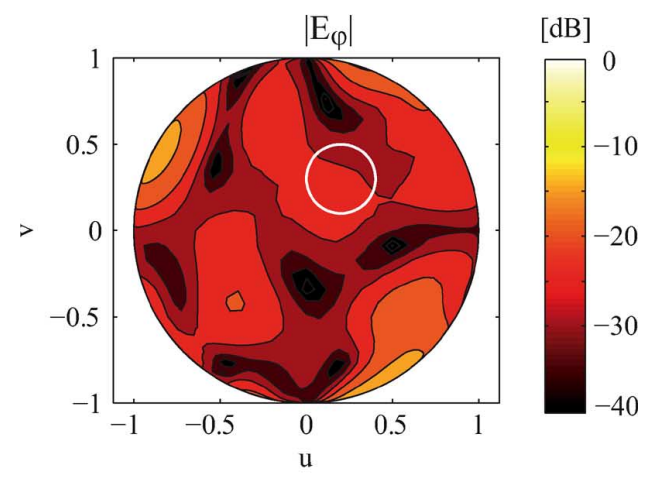

(c)

Fig. 9. Far field patterns corresponding to the linear polarization synthesis with the planar array shown in Fig. 6(a). (a) The magnitude of the total electric field is lower than $-10 \mathrm{~dB}$ outside the white circle. A linear polarization according to $\theta$ is optimized inside the white circle plotted in (b) and (c). In this region, $\left|E_{\varphi}\right|$ is at most $19 \mathrm{~dB}$ lower than $\left|E_{\theta}\right|$.

any geometry composed of elements that can have arbitrary and differing radiation patterns, can be handled and any state of polarization can be synthesized.

Using the polarization formulation proposed in [22], this synthesis problem has been written as a convex optimization problem, that requires to be iterated a small number of times. As opposed to other contributions, the desired polarization is optimized, not only in a single direction, but over a whole angular range, which is a frequently encountered requirement. The proposed synthesis method can easily be modified to yield an optimization problem that minimizes the side lobe levels, while guaranteeing a specified accuracy of the polarization over an angular range.

Various numerical examples of joint power pattern and polarization synthesis have been presented to validate the proposed 
approach and to show its potentialities. The design of a sequentially rotated array has first been considered to ascertain that the proposed approach recovers the expected weightings. Arbitrary linear and planar arrays as well as a conformal array have been synthesized to radiate both linear and circular polarization to show the flexibility of our approach. The mutual coupling effects between elements have been also taken into account in the synthesis procedure by applying the active element pattern method. Finally, the extension to the synthesis of two-dimensional power pattern with optimized polarization has been addressed to show the efficiency of the approach in terms of computation load.

\section{ACKNOWLEDGMENT}

The authors would like to thank the reviewers for their comments that helped improve the paper as well as M. Kupka Dias da Silva for fruitful discussions.

\section{REFERENCES}

[1] D. G. Giuli, "Polarization diversity in radars," Proc. IEEE, vol. 74, pp. 245-269, Feb. 1986.

[2] M. A. Sletten and D. B. Trizna, "An ultrawideband, polarimetric radar for the studies of sea scatter," IEEE Trans. Antennas Propag., vol. 42, no. 11, pp. 1461-1466, Nov. 1994.

[3] J. Vivekanandan, V. N. Bringi, M. Hagen, and P. Meischner, "Polarimetric radar studies of atmospheric ice particles," IEEE Trans. Geosci. Remote Sensing, vol. 32, no. 1, pp. 1-10, Jan. 1994.

[4] M. R. Andrews, P. P. Mitra, and R. De Carvalho, "Tripling the capacity of wireless communications using electromagnetic polarization," $\mathrm{Na}$ ture, vol. 409, pp. 316-318, Jan. 2001.

[5] W. C. Y. Lee and Y. S. Yeh, "Polarization diversity system for mobile radio," IEEE Trans. Commun., vol. 26, pp. 912-923, Oct. 1972.

[6] C. B. Dietrich, K. Dietze, J. R. Nealy, and W. L. Stutzman, "Spatial, polarization, and pattern diversity for wireless handheld terminals," IEEE Trans. Antennas Propag., vol. 49, no. 9, pp. 1271-1281, Sept. 2001.

[7] D. C. Cox, "Antenna diversity performance in mitigating the effects of portable radio telephone orientation and multipath propagation," IEEE Trans. Commun., vol. 31, no. 5, pp. 620-628, May 1983.

[8] R. T. Compton, Jr., "The tripole antenna: An adaptive array with full polarization flexibility," IEEE Trans. Antennas Propag., vol. 29, no. 6, pp. 944-952, Nov. 1981.

[9] A. J. Weiss and B. Friedlander, "Performance analysis of diversely polarized antenna arrays," IEEE Trans. Signal Processing, vol. 39, no. 7, pp. 1589-1603, Jul. 1991.

[10] A. Schell and A. Ishimaru, "Antenna pattern synthesis," in Antenna Theory, R. E. Collin and F. J. Zucker, Eds. New York: McGraw-Hill, 1969 , ch. 10 , pt. 1.

[11] W. L. Stutzman and G. A. Thiele, Antenna Theory and Design. New York: Wiley, 1981, ch. 10.

[12] L. I. Vaskelainen, "Iterative least-squares synthesis methods for conformal array antennas with optimized polarization and frequency properties," IEEE Trans. Antennas Propag., vol. 45, no. 7, pp. 1179-1185, Jul. 1997.

[13] C. Dohmen, J. W. Odendaal, and J. Joubert, "Synthesis of conformal arrays with optimized polarization," IEEE Trans. Antennas Propag., vol. 55, no. 10, pp. 2922-2925, Oct. 2007.

[14] H. Lebret and S. Boyd, "Antenna pattern synthesis via convex optimization," IEEE Trans. Signal Processing, vol. 45, no. 3, pp. 526-531, Mar. 1997.

[15] O. M. Bucci, M. D’Urso, and T. Isernia, "Exploiting convexity in array antenna synthesis problems," presented at the IEEE Radar Conf., Roma, Italy, May 2008.
[16] L. Caccavale, T. Isernia, and F. Soldovieri, "Methods for optimal focusing of microstrip array antennas including mutual coupling," IEE Microw. Antennas Propag., vol. 147, no. 3, pp. 199-202, Jun. 2000.

[17] J.-J. Xiao and A. Nehorai, "Optimal beam pattern synthesis of a polarized array," presented at the IEEE Statistical Signal Processing Workshop, Madison, WI, Aug. 2007.

[18] J.-J. Xiao and A. Nehorai, "Performance of beampattern synthesis using high-dimensional vector antenna arrays," presented at the Sensor, Signal and Information Processing Workshop, Sedona, AZ, May 2008.

[19] J.-J. Xiao and A. Nehorai, "Optimal polarized beampattern synthesis using a vector-antenna array," IEEE Trans. Signal Processing, vol. 57, no. 2, pp. 576-587, Feb. 2009.

[20] A. Ben-Tal and A. S. Nemirovski, "Lectures on modern convex optimization: Analysis, algorithms, and engineering applications," in Society for Industrial and Applied Mathematics. Philadelphia, PA: MPS-SIAM, 2001.

[21] G. A. Deschamps, "Part II-Geometrical representation of the polarization of a plane electromagnetic wave," Proc. IRE, vol. 39, pp. 540-544, May 1951.

[22] A. Nehorai and E. Paldi, "Vector-sensor array processing for electromagnetic source localization,” IEEE Trans. Signal Processing, vol. 42, no. 2, pp. 376-398, Feb. 1994.

[23] S. P. Boyd and L. Vandenberghe, Convex Optimization. Cambridge, U.K.: Cambridge Univ. Press, 2004.

[24] B. Fuchs and J. J. Fuchs, "Optimal narrow beam low sidelobe synthesis for arbitrary arrays," IEEE Trans. Antennas Propag., vol. 58, no. 6, pp. 2130-2135, Jun. 2010.

[25] SeDuMi [Online]. Available: http://sedumi.ie.lehigh.edu/

[26] T. Teshirogi, M. Tanaka, and W. Chujo, "Wideband circularly polarized array antenna with sequential rotations and phase shift of elements," in Proc. of ISAP, 1985, pp. 117-120.

[27] J. Huang, "A technique for an array to generate circular polarization with linearly polarized elements," IEEE Trans. Antennas Propag., vol. 34, no. 9, pp. 1113-1124, Sep. 1986.

[28] D. F. Kelley and W. L. Stutzman, "Array antenna pattern modeling methods that include mutual coupling effects," IEEE Trans. Antennas Propag., vol. 41, no. 12, pp. 1625-1632, Dec. 1993.

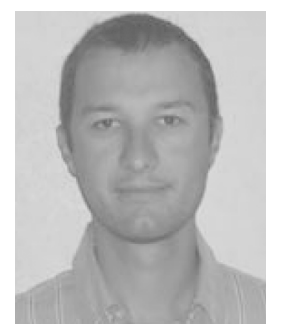

Benjamin Fuchs (S'06-M'08) received the Electronics Engineering degree and the M.S. degree in electronics from the National Institute of Applied Science (INSA) of Rennes, France, in 2004 and the Ph.D. degree from the University of Rennes 1, France, in 2007.

In 2008, he was a Postdoctoral Research Fellow at the Swiss Federal Institute of Technology (EPFL), Lausanne, Switzerland. In 2009, he joined the Institute of Electronics and Telecommunications of Rennes (IETR), as a Researcher at the Centre National de la Recherche Scientifique (CNRS). Since 2011, he is on leave at EPFL. His research interests include mode matching techniques, millimeter-wave antennas, focusing devices (lens antennas) and array synthesis methods.

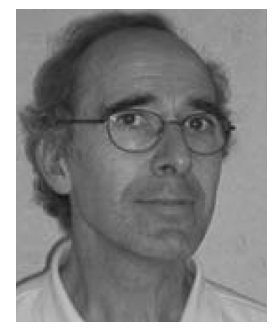

Jean Jacques Fuchs (M'81) was born in France in 1950. He graduated from the "École Supérieure d'Électricité" Paris, France, in 1973 and received the M.S. degree in electrical engineering from the Massachusetts Institute of Technology, Cambridge, in 1974.

After a short period in industry with Thomson-C.S.F., he joined the "Institut de Recherche en Informatique et Systèmes Aléatoires" (IRISA), France, in 1976. Since 1983, he is a Professor at the "Université de Rennes 1" France. His research interests shifted from adaptive control and identification, in which he obtained the "Thèse d'Etat" in 1982, towards signal processing. He is now involved in array processing and sparse representations. 